\title{
A RESULT ON NEARNESS OF FUNCTIONS AND THEIR REGULAR C-FRACTION EXPANSIONS
}

\author{
LISA JACOBSEN AND HAAKON WAADELAND
}

(Communicated by Irwin Kra)

\begin{abstract}
We shall prove a "nearness"-result of the following type. If $f(w)$ is a holomorphic function in $\Omega=\{w \in \mathrm{C} ;|\arg (1+4 w)|<\pi\}$ and "sufficiently near" the function $(\sqrt{1+4 w}-1) / 2$, then $f(w)$ has a regular $C$-fraction expansion $K\left(a_{n} w / 1\right)$ where $a_{n} \rightarrow 1$.
\end{abstract}

\section{THE RESUlT}

The present paper deals with regular C-fraction expansions

$$
\stackrel{\infty}{n=1}_{n}^{\infty}\left(\frac{a_{n} w}{1}\right)=\frac{a_{1} w}{1}+\frac{a_{2} w}{1}+\ldots+\frac{a_{n} w}{1}+\ldots,
$$

$a_{n} \in \mathbf{C}, a_{n} \neq 0$, of functions $f(w)$. It is well known that (1.1) corresponds to a formal power series

$$
d_{1} w+d_{2} w^{2}+\ldots+d_{n} w^{n}+\ldots,
$$

$d_{n} \in \mathbf{C}$. For definition of correspondence we refer to [2, Sec. 5.1, p. 148]. If (1.2) is the Taylor expansion of a function $f$ of a complex variable $w$, holomorphic in a neighborhood of the origin, we say that (1.1) is the regular Cfraction expansion of $f(w)$. To a formal power series there always corresponds a C-fraction [2, Cor. 5.3, p. 156], but not always a regular C-fraction (1.1). Necessary and sufficient conditions for such correspondence are known, see e.g. [2, Thm. 7.2, p. 223]. They are expressed in terms of Hankel determinants of the coefficients $d_{n}$, and are in general impractical in use. The very simplest (infinite) $\mathrm{C}$-fraction is

$$
\underset{n=1}{\infty}\left(\frac{w}{1}\right)=\frac{w}{1}+\frac{w}{1}+\ldots+\frac{w}{1}+\ldots .
$$

This C-fraction corresponds to the Taylor series expansion of

$$
\frac{1}{2}(\sqrt{1+4 w}-1), \quad \operatorname{Re} \sqrt{1+4 w}>0,
$$

Received by the editors February 29, 1988 and, in revised form, October 31, 1988.

1980 Mathematics Subject Classification (1985 Revision). Primary 30B70; Secondary 40A15, $41 \mathrm{~A} 20,41 \mathrm{~A} 21$.

Key words and phrases. Continued fractions, C-fractions, regular C-fractions, C-fraction expansions. 
i.e. to

$\left(1.4^{\prime}\right) \frac{1}{2} \sum_{k=1}^{\infty}\left(\left(-\frac{1}{2}\right)_{k}(-4 w)^{k}\right) / k !=w-w^{2}+2 w^{3}-5 w^{4}+14 w^{5}-42 w^{6}+\ldots$

Furthermore, the regular C-fraction (1.3) is known to converge to the function (1.4), uniformly on compact subsets of the complement $\Omega$ of the ray $(-\infty,-1 / 4]$, i.e.

$$
\Omega=\{w \in \mathbf{C} ;|\arg (w+1 / 4)|<\pi\} .
$$

(Keep in mind that the series $\left(1.4^{\prime}\right)$ diverges outside the circle $|w|=1 / 4$.)

The problem to be discussed in the present paper is, vaguely formulated, the following: If a function in some proper sense is "near" the function (1.4), will then a corresponding regular C-fraction exist, and will it be "near" the C-fraction (1.3)? Our particular answer to this question is expressed in the following:

Theorem 1.1. To any $R>2$ there is an $\varepsilon>0$, such that the following holds: Let $g$ be a function, holomorphic in the disk $|z|<R$, normalized by $g(0)=0$ and such that

$$
|g(z)| \leq \varepsilon \text { in }|z|<R .
$$

Then the function $f$, defined in the complement $\Omega$ of the ray $(-\infty,-1 / 4]$ by

$$
f(w)=\frac{\sqrt{1+4 w}-1}{2}\left(1+g\left(\frac{2 w}{1+2 w+\sqrt{1+4 w}}\right)\right), \quad \operatorname{Re} \sqrt{1+4 w}>0,
$$

has a regular $C$-fraction expansion $K\left(a_{n} w / 1\right)$, which is limit-periodic, $a_{n} \rightarrow 1$, and converges to $f(w)$ uniformly on compact subsets of $\Omega$.

Remark. As will be discussed later, the function

$$
z=\frac{2 w}{1+2 w+\sqrt{1+4 w}},
$$

being the inverse of the Koebe function, maps $\Omega$ one-to-one and conformally onto the open unit disk $U$. Hence $f(w)$ is well defined in $\Omega$.

Notice also the simple form of $(1.7), f(w)=(\sqrt{1+4 w}-1) / 2+R(w)$, where $R(w)$ has to be "small enough" in the sense mentioned. We see that $R(0)=R^{\prime}(0)=0$.

We first show two examples:

Example 1. With $g(z)=z / 3$ and thus

$$
f(w)=\frac{\sqrt{1+4 w}-1}{2}\left(1+\frac{2 w}{3(1+2 w+\sqrt{1+4 w})}\right)
$$

we find the Taylor expansion

$$
f(w)=w-\frac{2}{3} w^{2}+w^{3}-2 w^{4}+\frac{14}{3} w^{5}-12 w^{6}+33 w^{7}+\ldots, \quad|w|<\frac{1}{4} .
$$


Since $f(w)$ can be written

$$
f(w)={ }_{2} F_{1}\left(-\frac{1}{2}, 1 ; 2 ;-4 w\right)-1,
$$

it follows indeed that $f(w)$ has a regular C-fraction expansion $K\left(a_{n} w / 1\right)$ with $a_{1}=1, a_{2 n}=\left(2 n^{2}+n-1\right) /\left(2 n^{2}+n\right)$ and $a_{2 n+1}=\left(2 n^{2}+3 n\right) /\left(2 n^{2}+3 n+1\right)$. (Since all $a_{n}$ are positive it is even an S-fraction.) This continued fraction converges to $f(w)$ in $\Omega$, uniformly on compact subsets.

Example 2. With $g(z)=\sin (z / 10)$ we get

$$
f(w)=\frac{\sqrt{1+4 w}-1}{2}\left(1+\sin \left(\frac{w}{5(1+2 w+\sqrt{1+4 w}}\right)\right) .
$$

By straightforward computation we find that its Taylor expansion has the first terms

$$
f(w)=w-\frac{9}{10} w^{2}+\frac{17}{10} w^{3}+\frac{24601}{6000} w^{4}+\frac{67207}{6000} w^{5}+\ldots,|w|<\frac{1}{4},
$$

and the C-fraction expansion has the following start

$$
\begin{aligned}
& f(w)=\frac{w}{1}+\frac{(9 / 10) w}{1}+\frac{(89 / 90) w}{1}+\frac{(16003 / 16020) w}{1} \\
& +\frac{(28482539 / 28482540) w}{1}+\ldots .
\end{aligned}
$$

Hence it seems reasonable to believe that $f(w)$ has a limit-periodic, regular Cfraction expansion $K\left(a_{n} w / 1\right)$, where $0<a_{n} \rightarrow 1$ rather fast. In this particular case we can in fact prove that it has a regular $\mathrm{C}$-fraction expansion (indeed an S-fraction expansion) by using a result from Wall's book [9, p. 279].

Remark. Theorem 1.1 establishes the existence of an $\varepsilon$, but does not offer any value, since no value was given in what we shall state as our Theorem 2.1. By squeezing the proof of the latter, in particular the inequalities (2.8) and (2.9) in [6], it is easy to see that for any $M$ with

$$
1<M<\sqrt{R / 2}
$$

the following $\varepsilon$ works:

$$
\varepsilon=\min \left\{\frac{1-2 / R}{M}, 1-M \sqrt{2 / R}, \frac{2}{3} \frac{1}{M}\left(1-\frac{1}{M}\right)^{2}\right\} .
$$

With this $\varepsilon$-value, however, we can not "catch" the functions in Example 1 and 2.

Before proving the theorem we shall look at it in a wider context: For different types of continued fraction expansions an interesting (and possibly important) question is to relate "nearness" of functions to "nearness" of continued fraction expansions. This is a "continuity type" of question, although no proper topology has been introduced. The results so far seem to indicate that the easiest way is to pass from nearness of continued fractions to nearness of functions. The 
simplest results of this type are proved in [7] and [4]. In [7] is proved that general T-fractions $K\left(F_{n} z /\left(1+G_{n} z\right)\right)$, where $F_{n} \rightarrow 1$ and $G_{n} \rightarrow-1$ sufficiently fast, are expansions at 0 and $\infty$ of functions "near" $z$ and -1 , respectively. Also [4] contains results on T-fractions "near" $K(z /(1-z))$. In [4] is furthermore proved that C-fractions $K\left(a_{n} w / 1\right)$, where $a_{n} \rightarrow 1$ sufficiently fast, are expansions of functions "near" $(\sqrt{1+4 w}-1) / 2$, the nearness concept here including type and location of the branch point at $w=-1 / 4$. A result in the opposite direction, i.e. from nearness of functions to nearness of continued fraction expansions, is proved in [6] for general $\mathrm{T}$-fraction expansions. This result will be used in the proof of Theorem 1.1, and will be stated in the next section. An earlier, related result for special $\mathrm{T}$-fractions is proved in [5], and a result for PC-fractions is established in the recent paper [3]. Several attempts to prove a similar result for regular $\mathrm{C}$-fraction expansions of functions near $(\sqrt{1+4 w}-1) / 2$ have so far failed, the reason being that a proper type of nearness to this function, i.e. a nearness which is preserved by the generating linear fractional transformations, was not found.

Theorem 1.1 of the present paper is a rather special result of this type for C-fractions, but the many unsuccessful attempts call for modesty. The key to the solution is the transformation between $\mathrm{C}$-fractions and certain $\mathrm{T}$-fractions by using the Koebe function and its inverse, and the subsequent use of an already existing result for general T-fractions. Let it finally be mentioned, that the conclusion of Theorem 1.1 also holds if in $(1.7)(1+g(\ldots))$ is replaced by $(b+g(\ldots))$ for an arbitrary $b \neq 0$. Since $\varepsilon$ in that case will depend upon $b$, the start of the theorem would have to be: To any $R>2$ and any $b \neq 0$ there is an $\varepsilon>0, \ldots$. It is readily seen that if Theorem 1.1 holds with $\varepsilon=\varepsilon_{0}$, the modified version holds with $\varepsilon=|b| \varepsilon_{0}$. Another modification would be to replace, on the right-hand side of (1.7), $w$ by $a w, a \neq 0$, in which case the regular C-fraction would be limit-periodic with $a_{n} \rightarrow a$, and the domain of convergence will be the complement of the ray from $-1 /(4 a)$ to $\infty$ radially away from 0 .

\section{ORgANIZING THE TOOLS}

The proof itself is very short, but we need to know some "space-consuming facts", the first one being that to any general T-fraction

$$
K\left(\frac{F_{n} z}{1+G_{n} z}\right)=\frac{F_{1} z}{1+G_{1} z}+\frac{F_{2} z}{1+G_{2} z}+\ldots+\frac{F_{n} z}{1+G_{n} z}+\ldots, \quad F_{n} \neq 0,
$$

with $G_{n} \neq 0$ there corresponds a unique pair of formal Laurent series

$$
\left\{\begin{array}{l}
L(z)=c_{1} z+c_{2} z^{2}+\ldots+c_{n} z^{n}+\ldots(\text { Correspondence at } 0), \\
L^{*}(z)=c_{0}+c_{-1} z^{-1}+\ldots+c_{-n} z^{-n}+\ldots(\text { Correspondence at } \infty) .
\end{array}\right.
$$

The converse does not hold generally, but it holds under certain hard-to-checkdeterminant-conditions on the coefficients $c_{n}$. The simplest and most well 
known example is the general $\mathrm{T}$-fraction

$$
K\left(\frac{z}{1-z}\right)=\frac{z}{1-z}+\frac{z}{1-z}+\ldots+\frac{z}{1-z}+\ldots,
$$

which corresponds to the two formal power series

$$
\left\{\begin{array}{l}
L(z)=1 z+0 z^{2}+\ldots+0 z^{n}+\ldots \\
L^{*}(z)=-1+0 z^{-1}+\ldots+0 z^{-n}+\ldots
\end{array}\right.
$$

In this particular case it is also known that $\left(2.1^{\prime}\right)$ converges uniformly on compact subsets of $|z|<1$ to $z$, and uniformly on compact subsets of $|z|>1$ to -1 . This is also the reference case for the nearness theorems on general $\mathrm{T}$-fractions in [7, 8]. Nearness of general T-fractions is to $\left(2.1^{\prime}\right)$ and nearness of functions to $z$ and -1 . We shall need the following theorem from [6], phrased as in [7].

Theorem 2.1. Given $c_{1} \neq 0$, and let $r$ and $\rho$ be any two positive numbers such that

$$
r>\frac{2}{\left|c_{1}\right|}, \quad \rho<\frac{1}{2\left|c_{1}\right|} .
$$

Then there exist numbers $\alpha>0, \beta>0$, such that if $\left(L(z), L^{*}(z)\right)$ is a pair (2.2) of formal Laurent series with that particular $c_{1}$-value, then the following holds: If $L(z)$ represents a holomorphic function $L$ in $|z|<r$ and

$$
\left|L(z)-c_{1} z\right| \leq \alpha \quad \text { in }|z|<r,
$$

and $L^{*}(z)$ represents a holomorphic function $L^{*}$ in $|z|>\rho$ and

$$
\left|L^{*}(z)+1\right| \leq \beta \quad \text { in }|z|>\rho,
$$

then a corresponding general $T$-fraction exists, is limit-periodic with $F_{n} \rightarrow F \neq 0$ and $G_{n} \rightarrow-F$ as $n \rightarrow \infty$, and converges to $L(z)$ locally uniformly in $|z|<$ $1 /|F|$ and to $L^{*}(z)$ locally uniformly in $|z|>1 /|F|$.

Next we need to know how to transform a regular C-fraction to a general T-fraction: For a given regular C-fraction $K\left(a_{n} w / 1\right)$ we substitute

$$
w=\frac{z}{(1-z)^{2}}=: k(z) \quad \text { (the Koebe function) }
$$

and get, by an equivalence transformation

$$
\frac{a_{1 \frac{z}{(1-z)^{2}}}}{1}+\frac{a_{2 \frac{z}{(1-z)^{2}}}}{1}+\ldots \simeq \frac{1}{1-z}\left(\frac{a_{1} z}{1-z}+\frac{a_{2} z}{1-z}+\ldots\right),
$$

which, apart from the factor $1 /(1-z)$ in front, is a general $\mathrm{T}$-fraction. In case of convergence we also have equality in addition to equivalence. On the other hand, to any general $\mathrm{T}$-fraction of this particular type we get, by an equivalence transformation

$$
\frac{F_{1} z}{1-z}+\frac{F_{2} z}{1-z}+\ldots \simeq(1-z)\left(\frac{F_{1 \frac{z}{(1-z)^{2}}}}{1}+\frac{F_{2} \frac{z}{(1-z)^{2}}}{1}+\ldots\right)
$$


and, by substituting $w$ from (2.6):

$$
\left(1-k^{-1}(w)\right)\left(\frac{F_{1} w}{1}+\frac{F_{2} w}{1}+\ldots\right) .
$$

Since the function $k$ maps the open unit disk one-to-one onto the complement $\Omega$ of the ray $(-\infty,-1 / 4]$ of the negative real axis, and the outside of the unit circle onto a copy of $\Omega$, by $k(1 / z)=k(z)$, the function $k^{-1}$ can be written as:

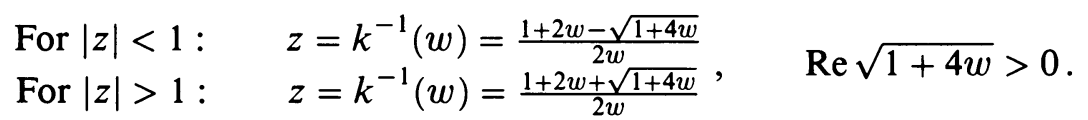

The simplest example of this C-T-bridge is the one between the continued fractions (1.3) and $\left(2.1^{\prime}\right)$ : With $w=k(z)$ in the open unit disk $U$ we find from (1.3), (1.4) and (1.5):

$$
(\sqrt{1+4 w}-1) / 2=\stackrel{W}{K}_{n=1}^{\infty}\left(\frac{w}{1}\right)=\stackrel{K}{K}_{n=1}^{\infty}\left(\frac{\frac{z}{(1-z)^{2}}}{1}\right)=\frac{1}{1-z}\left(\frac{z}{1-z}+\frac{z}{1-z}+\ldots\right) .
$$

We furthermore have

$$
\frac{1}{2} \sqrt{1+4 w}=\sqrt{\frac{z}{(1-z)^{2}}+\frac{1}{4}}=\frac{1}{2} \sqrt{\left(\frac{1+z}{1-z}\right)^{2}} .
$$

The case $|z|<1$. Since $\operatorname{Re}((1+z) /(1-z))>0$ for $z \in U$, we get from (2.11) that

$$
(\sqrt{1+4 w}-1) / 2=\frac{1}{2}\left(\frac{1+z}{1-z}\right)-\frac{1}{2}=\frac{z}{1-z},
$$

and $(2.10)$ gives

$$
z=\frac{z}{1-z}+\frac{z}{1-z}+\ldots \quad \text { for } z \in U .
$$

The case $|z|>1$. Since $\operatorname{Re}(-(1+z) /(1-z))>0$ for $|z|>1$ we get from (2.11) that

$$
(\sqrt{1+4 w}-1) / 2=\frac{1}{2}\left(-\frac{1+z}{1-z}\right)-\frac{1}{2}=\frac{-1}{1-z},
$$

and $(2.10)$ gives

$$
-1=\frac{z}{1-z}+\frac{z}{1-z}+\ldots \quad \text { for }|z|>1 .
$$

It is a routine matter to check that the transformation

$$
w=k(z), \quad z=k^{-1}(w)
$$

preserves correspondence at 0 . More precisely: If the regular $\mathrm{C}$-fraction $K\left(a_{n} w / 1\right)$ corresponds at $w=0$ to the formal power series

$$
d_{1} w+d_{2} w^{2}+d_{3} w^{3}+d_{4} w^{4}+\ldots,
$$


then the general T-fraction $K\left(a_{n} z /(1-z)\right)$ corresponds at $z=0$ to the formal power series

$$
\begin{aligned}
& (1-z)\left(d_{1} k(z)+d_{2} k(z)^{2}+d_{3} k(z)^{3}+d_{4} k(z)^{4}+\ldots\right) \\
& \quad=d_{1} z+\left(d_{2}+d_{1}\right) z^{2}+\left(d_{3}+3 d_{2}+d_{1}\right) z^{3}+\left(d_{4}+5 d_{3}+6 d_{2}+d_{1}\right) z^{4}+\ldots .
\end{aligned}
$$

Conversely, if the general T-fraction $K\left(F_{n} z /(1-z)\right)$ corresponds at $z=0$ to the formal power series

$$
c_{1} z+c_{2} z^{2}+c_{3} z^{3}+c_{4} z^{4}+\ldots,
$$

then the regular C-fraction $K\left(F_{n} w / 1\right)$ corresponds at $w=0$ to the formal power series

$$
\begin{aligned}
& \left(1-k^{-1}(w)\right)^{-1}\left(c_{1} k^{-1}(w)+c_{2} k^{-1}(w)^{2}+c_{3} k^{-1}(w)^{3}+c_{4} k^{-1}(w)^{4}+\ldots\right) \\
& \quad=c_{1} w+\left(c_{2}-c_{1}\right) w^{2}+\left(c_{3}-3 c_{2}+2 c_{1}\right) w^{3}+\left(c_{4}-5 c_{3}+9 c_{2}-5 c_{1}\right) w^{4}+\ldots .
\end{aligned}
$$

\section{Proof of the theorem}

Since $G_{n} \neq 0$ is sufficient for existence of a pair $\left(L, L^{*}\right)$ of corresponding power series, the existence is no problem for general $T$-fractions of the form $K\left(F_{n} z /(1-z)\right)$. We shall need the relation between $L$ and $L^{*}$ in this particular case.

Lemma 3.1. If

$$
L(z)=c_{1} z+c_{2} z^{2}+c_{3} z^{3}+\ldots
$$

is the corresponding series of the general $T$-fraction

$$
\frac{F_{1} z}{1-z}+\frac{F_{2} z}{1-z}+\frac{F_{3} z}{1-z}+\ldots
$$

at 0 , then

$$
L^{*}(z)=-c_{1}-c_{2} z^{-1}-c_{3} z^{-2}-\ldots
$$

is the corresponding series of (3.2) at $\infty$.

Proof. For $z \neq 0$ the continued fraction (3.2) is equivalent to the continued fraction

$$
\frac{-F_{1}}{1-\frac{1}{z}}+\frac{F_{2} \frac{1}{z}}{1-\frac{1}{z}}+\frac{F_{3} \frac{1}{z}}{1-\frac{1}{z}}+\ldots
$$

Remark. It is straightforward to prove, that if a pair $\left(L, L^{*}\right)$ of formal power series of the form

$$
\begin{gathered}
L(z)=c_{1} z+c_{2} z^{2}+c_{3} z^{3}+\ldots, \\
L^{*}(z)=-c_{1}-c_{2} z^{-1}-c_{3} z^{-2}-\ldots,
\end{gathered}
$$

i.e., $L^{*}(z)=-z L(1 / z)$, has a corresponding general T-fraction, then it is of the form (3.2). The crucial point in the argument is, that with

$$
L(z)=\frac{c_{1} z}{1-z+L_{1}(z)}, \quad L^{*}(z)=\frac{c_{1} z}{1-z+L_{1}^{*}(z)}
$$

we have $L_{1}^{*}(z)=-z L_{1}(1 / z)$. 
To prove Theorem 1.1 we shall simply apply Theorem 2.1 to a pair of functions with expansions (3.1a) and (3.1b) at 0 and at $\infty$ respectively. It is important to note that the pair $(\alpha, \beta)$ in Theorem 2.1 only depends upon $r, \rho$ and $c_{1}$, and that for a permitted pair $(\alpha, \beta)$, all $\left(\alpha^{\prime}, \beta^{\prime}\right)$ with $0 \leq \alpha^{\prime} \leq \alpha$, $0 \leq \beta^{\prime} \leq \beta$ work. In our case, if $L(z)$ represents a function, holomorphic in $|z|<r$, then

$$
L^{*}(z)=-z L\left(\frac{1}{z}\right)
$$

is holomorphic in $|z|>1 / r$, i.e. we choose $\rho=1 / r$, which is permitted if

$$
r>2 \max \left(\left|c_{1}\right|, \frac{1}{\left|c_{1}\right|}\right) .
$$

We choose in the following $c_{1}=1$, and let $r$ be any number $>2$. If

$$
|L(z)-z| \leq \alpha \quad \text { in }|z|<r,
$$

then by Schwarz' lemma

$$
\left|\frac{L(z)}{z}-1\right| \leq \frac{\alpha}{r} \quad \text { in }|z|<r,
$$

and hence

$$
\left|L^{*}(z)+1\right| \leq \frac{\alpha}{r} \quad \text { in }|z|>\frac{1}{r},
$$

which means that for the case

$$
L^{*}(z)=-z L(1 / z)
$$

we get the condition (2.5) in Theorem 2.1 "for free" when $\alpha$ is small enough. We thus have the following corollary to Theorem 2.1.

Corollary 3.2. To any $R>2$ there is an $\alpha>0$, such that the following holds: If

$$
L(z)=z+c_{2} z^{2}+c_{3} z^{3}+\ldots
$$

represents a holomorphic function in $|z|<R$, and

$$
|L(z)-z| \leq \alpha \quad \text { in }|z|<R,
$$

then a general T-fraction of the form

$$
\frac{F_{1} z}{1-z}+\frac{F_{2} z}{1-z}+\ldots+\frac{F_{n} z}{1-z}+\ldots
$$

exists, which corresponds to $L(z)$ at $z=0$. The continued fraction is limitperiodic with $F_{n} \rightarrow 1$, and converges to $L(z)$ uniformly on compact subsets of the unit disk $U$.

Remark. It is readily seen that $F_{1}=1$. With an arbitrary $c_{1} \neq 0$ instead of $c_{1}=1$ we can reduce to this case by studying $L(z) / c_{1}$ instead of $L(z)$. The boundedness condition is then

$$
\left|L(z)-c_{1} z\right| \leq \tilde{\alpha}=\left|c_{1}\right| \alpha
$$


and $F_{1}=c_{1}$. Hence, there is no restriction of generality to assume $c_{1}=1$. The only remaining step now is to translate this to a result on $\mathrm{C}$-fractions. From (2.7) and (2.8) we know that if $f(w)$ is holomorphic in a neighborhood of $w=0, f(0)=0$, and $f$ furthermore is such that

$$
L(z)=(1-z) f\left(\frac{z}{(1-z)^{2}}\right)
$$

satisfies the conditions of Corollary 3.2, then the conclusion holds. This in turn implies that $f(w)$ has a regular C-fraction expansion $K\left(F_{n} w / 1\right)$, which is limit-periodic, with $F_{n} \rightarrow 1$, and converges to $f(w)$ uniformly on compact subsets of $\Omega$. Put, with $L$ as in (3.4)

$$
L(z)-z=z g(z) \text {. }
$$

Since the boundedness condition in Corollary 3.2 is

$$
|z g(z)| \leq \alpha \text { in }|z|<R,
$$

we have from Schwarz' lemma

$$
|g(z)| \leq \frac{\alpha}{R}=\varepsilon .
$$

On the other hand, if $g$ is holomorphic in $|z|<R, g^{\prime}(0)=0$ and $\left(3.6^{\prime}\right)$ holds, then also (3.6) holds, and the Taylor expansion of $z g(z)$ starts with $z$. From (3.4) and (3.5) we find how to express $f$ in terms of $g$ :

$$
f\left(\frac{z}{(1-z)^{2}}\right)=\frac{z}{1-z}(1+g(z)) .
$$

The substitution of $z=k^{-1}(w)=2 w /(1+2 w+\sqrt{1+4 w})$ then concludes the proof of Theorem 1.1.

Remark. The simplest example, except for the trivial one $(g(z) \equiv 0)$ is

$$
g(z)=\frac{1}{q} z
$$

\begin{tabular}{|c|c|c|c|c|c|c|c|c|c|}
\hline & & & 2 & & & & & & \\
\hline$q=4$ & $a_{n}$ & 1 & $\frac{3}{4}$ & $\frac{11}{12}$ & $\frac{32}{33}$ & $\overline{88}$ & $\frac{231}{232}$ & & \\
\hline$q=3$ & $a_{n}$ & 1 & $\frac{2}{3}$ & 5 & & $\frac{14}{15}$ & & & \\
\hline$=-1$ & $a_{n}$ & 1 & 2 & $\overline{2}$ & & $\frac{2}{3}$ & $\frac{4}{3}$ & & \\
\hline$=-2$ & $a$ & & & 5 & & & $\frac{105}{104}$ & $\frac{272}{273}$ & \\
\hline
\end{tabular}

where $q$ is a constant. Example 1 is such a case, with $q=3$. By using formulas, strongly indicated by MACSYMA, one can prove that for $q$ not on the interval $(-1,3)$ of the real axis the function $f(w)$ in $(1.7)$ has a regular C-fraction expansion $K\left(a_{n} w / 1\right)$ where $a_{n} \rightarrow 1$ when $n \rightarrow \infty$. An illustration with some real, "permitted" $q$-values is shown below: 


\section{FinAl COMMENTS}

The condition $R>2$ is inherited from Theorem 2.1, which in turn inherited it from the result on special T-fractions in [5]. Hovstad was able to push it down to $R>1$ for special T-fractions [1], and in [8] this was also done for general $\mathrm{T}$-fractions in two very special cases. It is likely that also in the present case the condition can be made weaker, perhaps down to $R>1$. Another interesting question would be to find explicitly a "good" $\varepsilon$ in terms of $R$. (See also (1.8).) Another natural question is to investigate $C$-fraction expansions of functions near something else than $(\sqrt{1+4 w}-1) / 2$, e.g. functions having other certain well known regular $\mathrm{C}$-fraction expansions.

\section{REFERENCES}

1. R. M. Hovstad, Solution of a convergence problem in the theory of T-fractions, Proc. Amer. Math. Soc. 48 (1975), 337-343.

2. W. B. Jones and W. J. Thron, Continued fractions: analytic theory and applications, Encyclopedia of Mathematics and its Applications Vol. 11, Addison-Wesley, Reading, Mass. 1980, distributed now by Cambridge University Press, New York.

3. O. Njästad and H. Waadeland, Strongly bounded holomorphic functions and PC-fractions, submitted

4. W. J. Thron and H. Waadeland, Analytic continuation of functions defined by means of continued fractions, Math. Scand. 47 (1980), 72-90.

5. H. Waadeland, On T-fractions of functions holomorphic and bounded in a circular disk, Norske Vid. Selsk. Skr. Trondheim, No. 8 (1964), 1-19.

6. _ On general T-fractions corresponding to functions satisfying certain boundedness properties, J. Approx. Theory 26 (1979), 317-328.

7. _ On limit-periodic general T-fractions and holomorphic functions, J. Approx. Theory 27 (1979), 329-345.

8. _ General T-expansions of bounded functions, Mathematics no. 2 (1983), Univ. of Trondheim.

9. H. S. Wall, Analytic theory of continued fractions, Van Nostrand, New York, 1948.

10. L. Jacobsen, and $\mathrm{H}$. Waadeland, When does $f(z)$ have a regular $C$-fraction expansion or a normal Padé table, to appear in Journ. of Comp. and Appl. Math. NORWAY

Division of Mathematical Sciences, University of Trondheim, N-7034 Trondheim, INSTITUte for Mathematics and Statistics, University of Trondheim, N-7055 DragVoll, NoRWAY 